\title{
Design Thinking : le design en tant que management de projet
}

Jean-Patrick Péché, Fabien Mieyeville, Renaud Gaultier

Propos de l'article :

$\checkmark$ Le Design Thinking, héritier du Design Industriel

$\checkmark$ Le Design Thinking, au-delà du terme hype, un outil opérationnel de management du projet

$\checkmark$ Le Design Thinking pour le déploiement de l'innovation

Dans un monde où le contexte économique est difficile et où les modèles économiques traditionnels sont malmenés, le Design Thinking semble être devenu une des voies de redressement les plus prometteuse, à tel point qu'il n'existe pas de grande école (aussi bien ingénieur que commerce) qui n'intègre pas cette notion dans son cursus d'apprentissage. Au delà de cet effet de mode, le Design Thinking est un mode de pensée dont les origines remontent au XIXème siècle, héritier du design industriel qu'un designer de talent appelé Tim Brown a remis sur le devant de la scène.

Dans cet article nous allons présenter ce qu'est le Design Thinking et la manière dont il peut s'intégrer dans tout processus d'innovation.

\section{LE PROCESSUS DE}

\section{CREATION/INNOVATION/DEVELOPPEMENT}

Si traditionnellement le processus de création/innovation/développement est celui de la Figure 1, Bill Buxton (Buxton 2007) met en évidence la nécessité de mixer au plus tôt les trois métiers du design, de l'ingénierie et du marketing afin de rendre le processus plus efficace et plus performant.

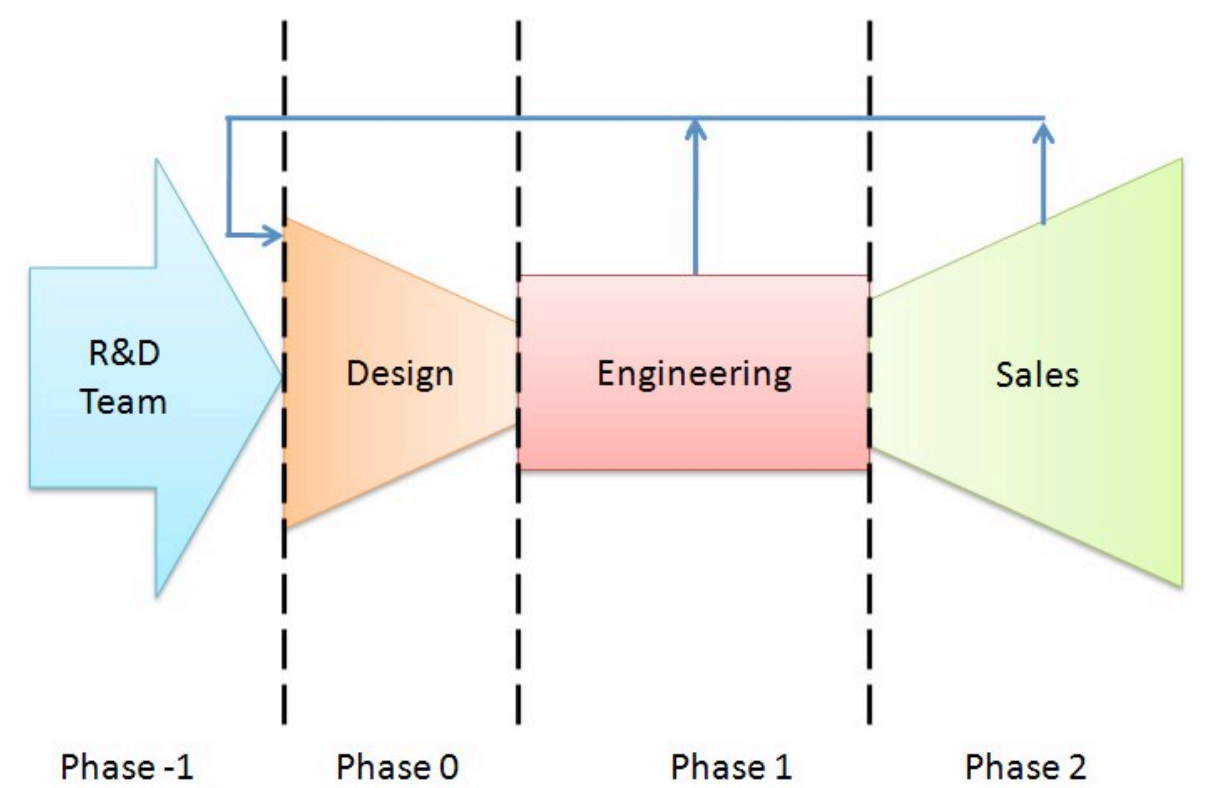

Figure 1 : Séquencement des phases et des expertises dans le processus de création/développement d'artefacts (Buxton, 2007)

Il est en effet nécessaire de briser l'approche en silo traditionnelle et de mixer au plus tôt les différentes compétences comme le montre la figure suivante (qui a de nombreuses similitudes avec l'approche préconisée par Tim Brown comme on le verra plus tard). Le modèle de formation des ingénieurs et du marketing et management repose actuellement sur le modèle de la Figure 1 et tendent à migrer vers le modèle de la Figure 2 au travers des différentes initiatives actuellement observables sur l'intégration de l'innovation dans ces cursus. 
Cette proposition de représentation de l'ordre d'entrée dans le projet des différentes catégories métier et de leur niveau de congruence de Buxton doit être étendue dans notre monde actuel où les réseaux sociaux et les relations sociales et sociétales deviennent prépondérantes. Il est désormais vital d'intégrer les sciences humaines et sociales à toutes les étapes de développement d'un produit/service. Un nombre croissant d'entreprises $(\mathrm{SEB}, \ldots)$ intègrent désormais des anthropologues dans leurs équipes de développement.

Ainsi, pour illustrer le modèle que nous proposons dans la Figure 2, les apports en sciences humaines peuvent être multiples : apports d'un philosophe dans l'accompagnement du travail de veille élargie ou ceux d'un anthropologue sur l'observation et les études des usages en phase 0 , intervention de l'ergonome pour définir les usages ou accompagnement d'un sémiologue pour accompagner le travail de codification formel en phase 1 et enfin retours possibles du philosophe en phase 2 pour la mise en cohérence du discours marketing avec les valeurs contenue dans la proposition finale...

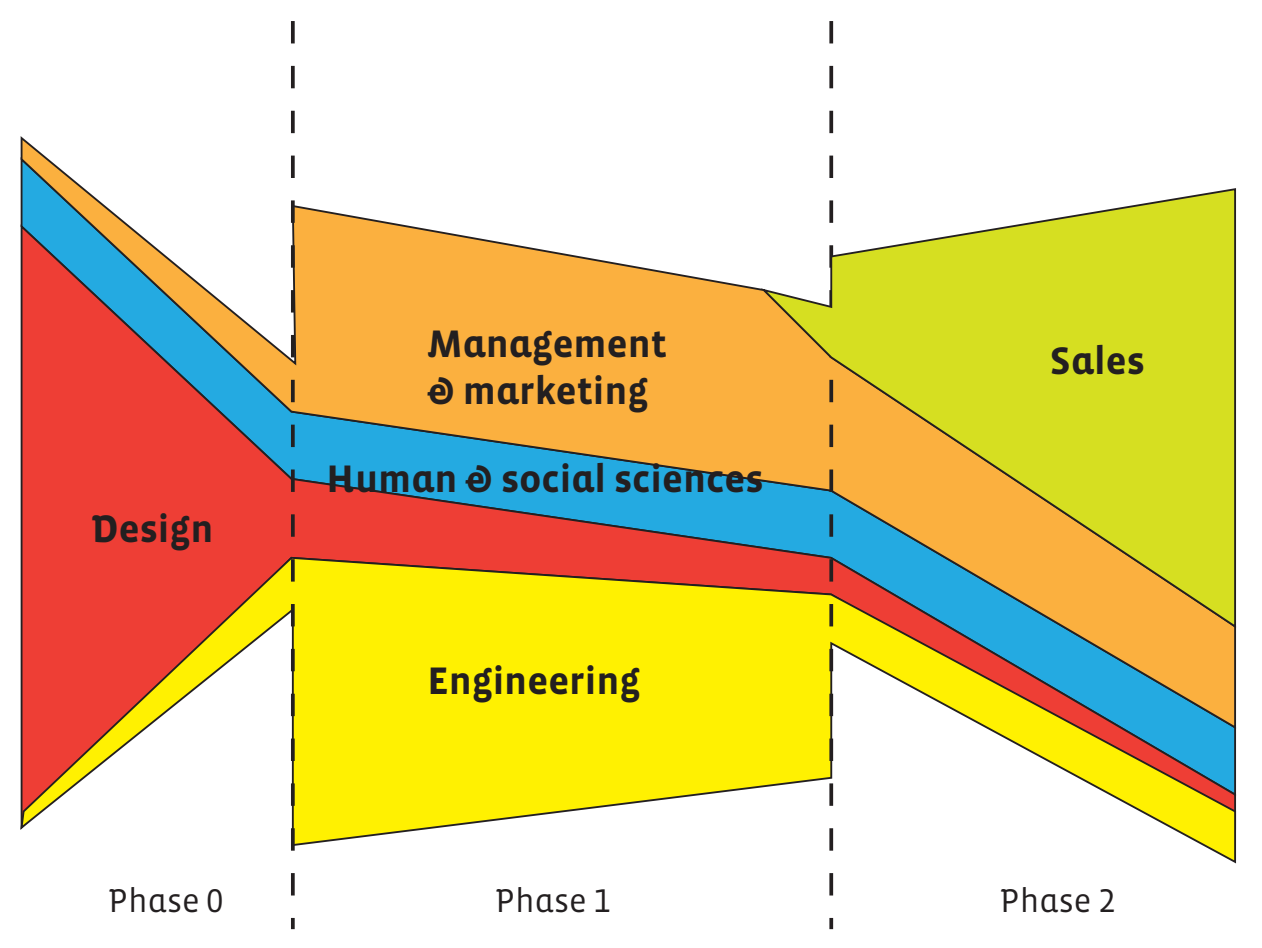

Figure 2 : Séquencement des phases dans le processus de création/développement d'artefact brisant le modèle en silo inspiré du modèle de Bill Buxton

Afin de parvenir à l'approche préconisée, il faut décloisonner les approches et les processus traditionnellement structurés en silo. Le Design Thinking en tant que méthode de management de projet permet cette interpénétration des différents métiers.

Après un bref historique du Design Thinking et un rapide aperçu du Design Thinking tel qu'il est perçu et enseigné aujourd'hui nous présenterons notre approche du Design Thinking en tant que méthode de management du projet. Nous montrerons également en quoi cette approche s'adapte à tout type de formation avant de conclure.

\section{DESIGN THINKING : ÉTAT DE L'ART}

De l'ère artisanale à l'ère industrielle, et la mondialisation de la production et de la consommation, une (très ) brève histoire du design 
Pour comprendre le développement du design depuis le développement de l'industrie dans la seconde moitié du $19^{\mathrm{e}}$ siècle jusqu'à nos jours, nous proposons quelques « balises » dont le choix est fondé sur l'importance créative, historique et économique (cf. encadré).

L'avènement de l'industrie a rompu la relation directe réalisateur/utilisateur de l'époque artisanale, générant ainsi le découplage des fonctions de conception, de fabrication, de commercialisation, puis découplant les fonctions de conception entre ingénieur et le créateur de modèle. L'industrie a d'abord principalement copié des modèles artisanaux avant de comprendre l'intérêt de se doter de ses propres formes (ou modèles) pour intégrer de manière plus efficace des contraintes liés aux outils de production, et à l'évolution des goûts et des usages de la société. Le design a ainsi évolué jusqu'à s'étendre au management de l'innovation à un niveau stratégique. Cette extension appelée Design Thinking est une culture née dans la Silicon Valley, au milieu des années 2000 promue par l'ingénieur David Kelley et le designer Tim Brown à la tête d'IDEO.

\section{RAPIDE HISTORIQUE DU DESIGN THINKING}

Créer le lien entre art et industrie : Arts and Crafts, mouvement fondé par John Ruskin et William Morris en Angleterre, 1853, 1890.

Ce mouvement est né d'une révolte de son contre l'industrialisation massive jugée dangereuse pour l'environnement, et productrice de médiocrité esthétique et sociale.

Ce mouvement d'abord moralisateur, a ensuite défini les fondements du design et préparé la venue du mouvement «Art Nouveau » qui a eu une influence dans toute l'Europe.

Établir des fondamentaux théoriques : le Bauhaus. Cet institut des arts et métiers fondé en 1919 à Weimar, par Walter Gropius. Son activité couvre l'architecture, les arts plastiques, est considérée comme précurseur du design contemporain. Fermé en 1933 par les nazis, cette école était surtout une école de pensée qui se posait la question de ce qu'est la création dans la société moderne et l'industrie. Elle a ainsi, par ce travail de théorisation, permis le développement des écoles de design.

Libérer la pensée créatrice : le mouvement pop. Milieu années 1960 à milieu années 1970. À partir du début des années 1960, de puissants mouvements créatifs et de contestation sociale voient le jour. Tous les domaines de la société sont concernés, les carcans explosent libérant ainsi de nouvelles pensées créatives dont la critique de la société de consommation. Ce puissant mouvement quasi mondial a permis de " penser autrement», en tenant compte davantage de modes d'usages « réels », sans inhibition sociale, générant ainsi de nouvelles approches « en rupture » favorisant l'innovation.

Affirmer le design comme mode de management de l'innovation à un niveau stratégique : le design thinking. Du milieu années 1970 à nos jours, la Silicon Valley. À cette époque, après la " révolution » pop, de grandes agences de design de renommée internationale se sont développées dans la région de Palo Alto, portées un contexte académique et de recherche (l'Université de Stanford par exemple), un contexte industriel forts, lié au développement de l'électronique et de l'informatique. L'importance stratégique de l'intégration du design dans le développement de cette nouvelle industrie a conduit à des stratégies de design ambitieuses (Apple, Google, etc.), le design est établi comme un vecteur stratégique de développement, et d'accompagnement du changement. Né à l'Université de Stanford en Californie avec Tim Brown et incarné par des sociétés telle IDEO le design thinking est un processus participatif de réflexion, d'action, et de résolution de problèmes, qui tient compte du contexte culturel, social et économique.

Cette méthode est aujourd'hui reconnue comme une des plus performante pour des projets d'organisation sociale, d'innovation de rupture, de développement d'activités dans un cadre de développement durable. Si le design thinking n'en est qu'à ses balbutiements en Amérique du Nord, il fait d'ores et déjà partie du quotidien des entreprises en Finlande. «Avec le design thinking, au lieu de se demander comment résoudre un problème, on se demande pourquoi on a ce problème. Rien que ce changement d'approche suffit à trouver des idées neuves», dit Mikko Kämäräinen, PDG de l'agence finlandaise de création Provoke.

Le design thinking, une « non-innovation » ? L’idée que la pratique du projet incluant le design dans un contexte pluridisciplinaire doit être favorisée n'est certes pas nouvelle, ni le centrage sur l'humain de la démarche du designer. « les premières théories du XIXe siècle, le design a été considéré comme le 
moyen d'humaniser la technique...»( Le Bœuf 2011). D'autre part le niveau de réussite des démarches de type design thinking est parfois remis en question (Nussbaum 2011), pendant que d'autres pensent que l'approche du Design Thinking est favorable à la réussite de projets de start-up notamment dans le domaine technologique (Witmeur 2012) et les contextes complexes.

\section{LE DESIGN THINKING EN GENERAL}

Le Design Thinking a donné lieu à de nombreuses formalisations et travaux théoriques qui peuvent être résumés de manière synthétique dans le tableau suivant extrait des travaux de Lucy Kimbell (Tableau 1).

Tableau 1 : Synthèse des différentes approches du Design Thinking (Kimbell 2011)

\begin{tabular}{|l|l|l|l|}
\hline & $\begin{array}{l}\text { Design Thinking en tant } \\
\text { que style cognitif }\end{array}$ & $\begin{array}{l}\text { Design Thinking en tant } \\
\text { que théorie du design }\end{array}$ & $\begin{array}{l}\text { Design Thinking en tant } \\
\text { que ressource } \\
\text { organisationnelle }\end{array}$ \\
\hline Focus & $\begin{array}{l}\text { Designer individuel, } \\
\text { principalement expert }\end{array}$ & $\begin{array}{l}\text { Design en tant que } \\
\text { discipline }\end{array}$ & $\begin{array}{l}\text { Entreprises et leurs } \\
\text { organisations en } \\
\text { recherche d'innovation }\end{array}$ \\
\hline Objectif du design & Résolution de problème & $\begin{array}{l}\text { Maîtrise des problèmes } \\
\text { épineux }\end{array}$ & Innovation \\
\hline Concepts clé & $\begin{array}{l}\text { Aptitude au design en } \\
\text { tant que forme } \\
\text { d'intelligence : réflexion } \\
\text { dans l'action, abduction }\end{array}$ & $\begin{array}{l}\text { Le design n'a pas de } \\
\text { spécificité ou de pratique } \\
\text { privilégiée }\end{array}$ & $\begin{array}{l}\text { Visualisation, } \\
\text { prototypage, empathie, } \\
\text { pensée intégratrice, } \\
\text { abduction }\end{array}$ \\
\hline Nature du problème & $\begin{array}{l}\text { Les problèmes sont mal } \\
\text { formulés, le problème et } \\
\text { la solution co-évoluent }\end{array}$ & $\begin{array}{l}\text { Les problèmes sont des } \\
\text { problèmes épineux }\end{array}$ & $\begin{array}{l}\text { Les problème sont des } \\
\text { problèmes } \\
\text { organisationnels }\end{array}$ \\
\hline $\begin{array}{l}\text { Sites de l'expertise du } \\
\text { design et de son activité }\end{array}$ & $\begin{array}{l}\text { Disciplines du design } \\
\text { traditionnelles }\end{array}$ & $\begin{array}{l}\text { Les quatre piliers du } \\
\text { design }\end{array}$ & $\begin{array}{l}\text { N'importe quel contexte } \\
\text { de l'accès au soin à l'eau } \\
\text { propre }\end{array}$ \\
\hline Source Kimbell, Lucy. “Rethinking Design Thinking: Part 1.” Design and Culture 3, no.3 3 (2011):285-306.
\end{tabular}

Lorsque le design thinking a émergé il y a de cela une décade, il a permis de repositionner les designers au centre du projet, non plus comme spécialistes de la forme mais aussi comme médiateur des cultures interdisciplinaires ou comme liant dans les équipes pluridisciplinaires. Dans ces cinq dernières années, avec les travaux de Tim Brown, ce terme est devenu plus englobant en plaçant l'être humain au centre de l'approche de résolution des problèmes en contraste avec les approches centrées sur les technologies ou les organisations traditionnellement adoptées jusque là.

\section{LE DESIGN THINKING SELON TIM BROWN}

Il n'est pas possible actuellement de discuter du design thinking sans évoquer même brièvement les travaux de Tim Brown qui a contribué à ramener au premier plan cette notion.

Après la conférence qu'il a tenue en décembre 2007 à Stanford «Strategy by Design: How Design Thinking Builds Opportunities » et après qu'il ait constaté un peu plus tard des tentatives de récupération par des écoles de management de « la pensée design » en créant des masters de design thinking (sans

\footnotetext{
${ }^{1}$ « Wicked problem » : théorie formalisée par Horst Rittel qui recouvre une classe de problèmes relatifs aux systèmes sociaux ou culturels qui sont mal formulés, où l'information est source de confusion, intégrant de nombreux clients et décideurs avec des valeurs contradictoires et où les ramifications dans l'ensemble du système sont profondément déroutantes.
} 
pour autant y intégrer des designers), Tim Brown a écrit son livre « Change by design » en 2009. La " pensée design » est ainsi portée par les designers! Le PDG fondateur d'un des plus grands réseaux internationaux d'agences de design, ou d'innovation, IDEO, consacre une part importance de son temps en conférences, écrits, et participe au développement de cette théorie avec Stanford, et publie régulièrement avec 'autres institutions (Harvard Business revue par exemple.

Le design thinking selon Tim Brown peut se résumer par la Figure 3 désormais célèbre extraite de son livre (Brown 2009).

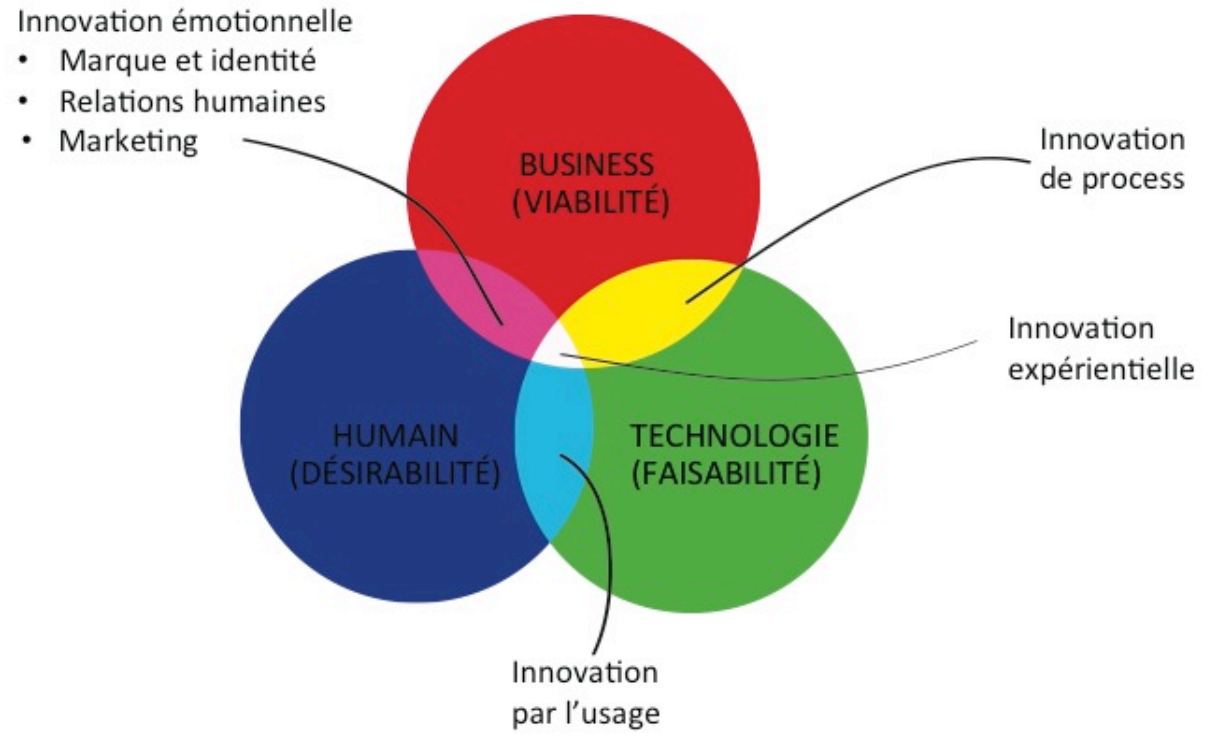

Figure 3 : le Design Thinking selon Tim Brown.

Ainsi la complexité de cette pensée et l'étendue des divers modes de réflexion utilisés sont ainsi mieux identifiés, et donc plus facilement partageables et modélisables.

Le travail de Tim Brown, de ses prédécesseurs, et de ses commentateurs, permet de réaffirmer l'efficacité de ce mode de réflexion, notamment en termes :

- d'efficience des métiers de création dans les processus d'innovation

- $\quad$ de pertinence du leadership dans le projet par le design, et au-delà de l'exercice du projet : mais aussi dans celui de l'entreprise, cette question est sans cesse reposée, mais de manière de plus en plus prégnante

- $\quad$ de prise en compte des éléments « subjectifs et intuitifs » (ou valeurs d'identité, ou de séduction, ou émotionnelles, ces éléments seront développés ensuite), en rapport avec une stratégie d'entreprise qui ne peut plus se battre que sur la seule technologie qui est de plus en plus standard, et donc de moins en moins « différenciante ».

Le design thinking se veut être l'approche de l'innovation par les usages tout en maintenant une prise en compte des aspects de faisabilité et de viabilité. Il n'est donc pas l'apanage des seuls designers : ces derniers sont culturellement mieux armés à l'intégration de ce mode de pensée mais cela peut être intégré par tout le monde.

Cependant l'approche proposée par Tim Brown et faisant désormais office de référence dans le monde des entreprises aussi bien qu'universitaire présente deux faiblesses. La première est l'absence d'information ou de structuration qui permet d'utiliser de manière concrète le Design Thinking comme outil de prise de décision : chez Tim Brown, la décision est le fruit des réflexions du designer après intégration du contexte et de l'ensemble des données des 3 sphères (Figure 3). La seconde est l'absence de temporalité dans la représentation qu'en fait Tim Brown alors que le Design Thinking accompagne un processus au travers de phases successives dans lequel, 
de plus, chaque sphère a une pondération plus ou moins élevée selon l'avancement du projet (en conjonction avec le diagramme initial de la Figure 2).

Nous proposons ici une extension du modèle du Design Thinking tel que présenté par Tim Brown qui permet d'inclure ces deux notions.

\section{LE DESIGN THINKING EN MANAGEMENT DE PROJET}

Comme développée en introduction, une innovation est un processus qui se décrit en un certain nombre de phases. Aux trois phases proposées par Bill Buxton, nous substituons un modèle en 5 phases inspiré du modèle du Design Toolkit educator ${ }^{2}$.

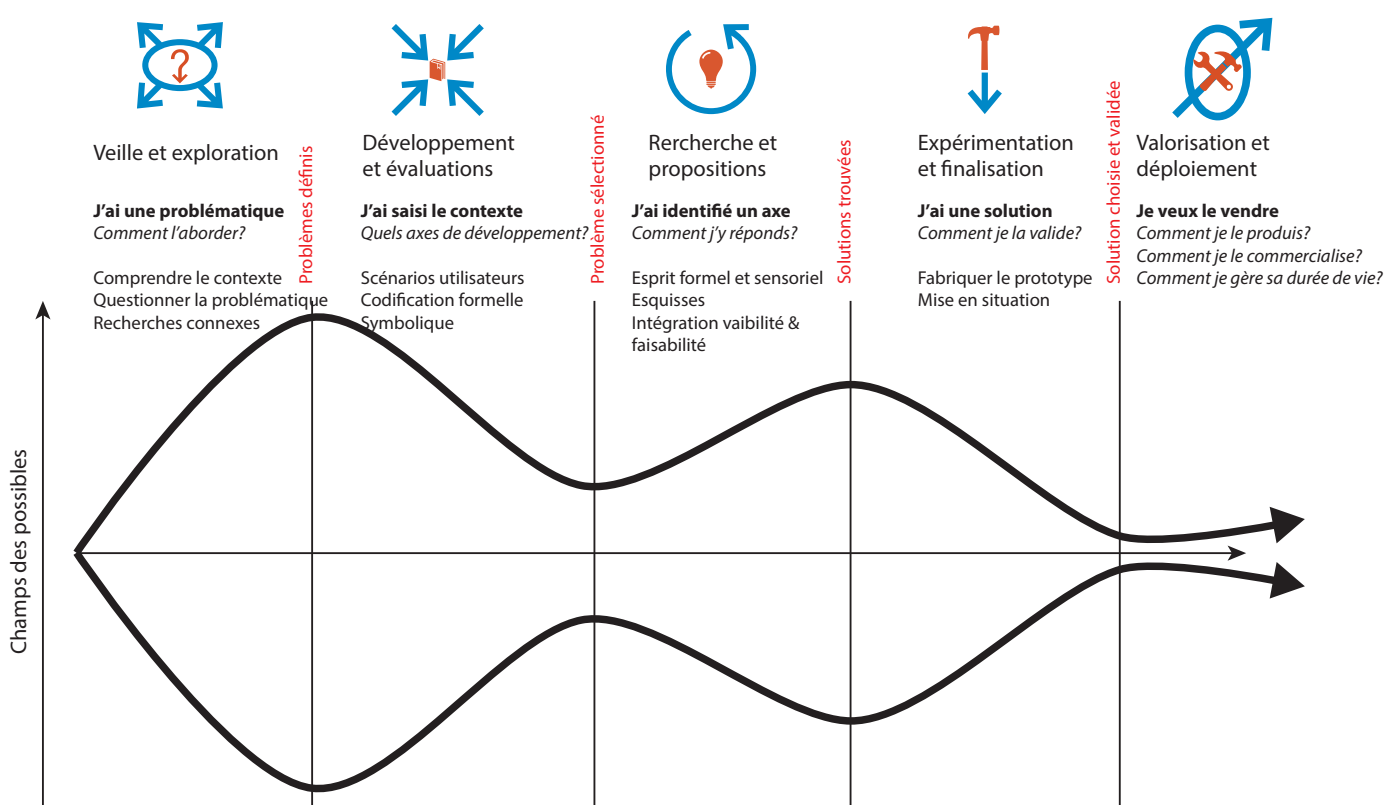

Figure 4 : Le Design Thinking, un processus.

La forme de cette courbe illustre les différentes successions de phases de divergence et de convergence dans la production et le développement des idées par le design.

\section{LE DESIGN THINKING : UNE APPROCHE SEQUENCEE}

Un projet se construit au travers de 5 phases, chacune d'entre elle ayant un objectif, des délivrables et sollicitant certains schémas cognitifs plutôt que d'autres.

Ces schémas cognitifs (qui fonctionnent souvent par paire) sont les suivants : Analyse/Synthèse, RéflexionObservation/Action - Réalisation, Convergence/Divergence, Concret/Abstrait

Les cinq phases du mode projet tel que perçu au travers du Design Thinking sont les suivantes : Veille et exploration, Recherche et propositions, Développement et évaluations, Expérimentations et finalisations et Valorisation et déploiement.

\section{Phase 1 : Veille et exploration}

En partant du postulat (récurrent en Design Thinking - cf. Tableau 1-) que tout problème est par nature maldéfini, le design thinking part de l'énoncé d'une « problématique » qui doit être étudiée de points de vue multiples, contextualisée puis confrontée à de multiples champs disciplinaires et culturels afin d'en dégager des formulation de problèmes ${ }^{3}$ à résoudre. Cette phase est une phase de veille et d'exploration dans laquelle on

\footnotetext{
${ }^{2} \mathrm{http}: / /$ www.designthinkingforeducators.com/design-thinking

${ }^{3}$ On peut préférer à la notation de problème la notion d'opportunité.
} 
sollicite plutôt des compétences de type analyse, recherche/observation qui trouve son ancrage dans le concret et le monde du réel en très forte divergence puisqu'il s'agit d'ouvrir le champ des possibles au maximum. Les activités principales visent à se doter de connaissances et ouvrir la créativité et permet de positionner l'équipe projet par rapport à la stratégie et aux objectifs du demandeur, d'identifier et adapter des choix méthodologiques, et de redéfinir les objectifs/besoins initialement exprimés. Les champs disciplinaires invoqués sont : Design, Sciences humaines et sociales (philosophie, sociologie, anthropologie), Sciences du commerce (marketing), Art (peinture, sculpture, architecture, musique, danse, design), Culture, Sciences techniques, Sociétés et cultures, Biologie,...

\section{Phase 2 : Recherche et propositions}

Cette phase est la phase au cours de laquelle on interprète le résultat de la veille et exploration. On extrait au cours de cette phase les axes de développement (ou « concepts design ») du projet. Cette phase est globalement convergente (on peut élargir ponctuellement les champs de possibilité pour étudier les ramifications d'un axe potentiel) puisqu'on restreint le champ des possibles à quelques axes après maturation et décantation de la phase précédente. On reste principalement dans l'analyse d'un contexte et de ses champs connexes tout en commençant une phase de synthèse qui va permettre le raffinement des opportunités et l'intégration des aspects sociaux, culturels, législatifs et économiques. Cette phase demeure réflexive mais définitivement dans l'abstrait puisqu'on esquisse des concepts, des codifications formelles et des schémas d'usages. Les principaux acteurs disciplinaires mobilisés sont : Design, Ergonomie et utilisateurs, Anthropologie, Techniciens, Marketing avancé, Plasticiens

\section{Phase 3 : Développement et évaluations}

Une fois les opportunités définies et les axes de développement du projet établis, la phase de « résolution du problème » à proprement parler débute. De manière similaire à la première phase, une exploration globalement divergente est menée de manière à faire émerger un très grand nombre de réponses possibles basées non plus sur les champs connexes mais ancrés dans les domaines techniques et financiers par validation des choix techniques et technologiques en regard avec les objectifs financiers. Cette phase de synthèse de solutions cohabite avec une analyse du contexte technologique et financier. C'est le temps des esquisses, des schémas conceptuels de solutions couplés avec leurs scénarios d'usage, du développement de l'esprit formel et sensoriel des concepts et des premiers maquettages grossiers. A la fin de cette phase deux à trois axes design devront être retenus. Les principaux champs disciplinaires mobilisés sont : Design, Ergonomie et utilisateurs, Ingénierie de conception et de production, marketing, sémiologues.

\section{Phase 4 : Expérimentation et finalisation}

Cette phase recouvre la définition précise de la solution proposée, sa fabrication sous la forme d'un démonstrateur ou prototype et sa validation en termes d'usages, de technologies, d'éthique, de viabilité financière et d'identité. Cette phase est la phase de prototypage, fabrication et réalisation, convergent vers la solution finale, synthèse de toutes les phases précédentes. On y démontre le respect des objectifs, du positionnement et de la stratégie design. Le projet présenté en fin de phase doit être directement transposable en production. Le prototype s'accompagne de plans techniques, de dessins numériques $2 \mathrm{D}$ et 3D, de maquette fonctionnelle ou de volume en couleur et de story-board de mode d'usage. Les disciplines concernées sont : Design, Ingénierie de conception et de production, philosophes et Marketing.

\section{Phase 5 : Valorisation et déploiement}

Cette phase regroupe la production et le déploiement commercial. Cette dernière phase permet de s'assurer que le design retenu est bien celui mis en fabrication ou exploitation et que les investissements et les choix techniques pour la mise en production sont respectés. Les champs disciplinaires mobilisés sont principalement ceux du marketing et de la production avec l'appui du design et de la conception.

\section{TEMPORALITE DU PROJET}

Comme on peut le constater, cette approche du design thinking en tant que management du projet possède certaines caractéristiques. Tout d'abord, elle peut être mise en application par tout corps de métier même si le designer, de par sa polyvalence sera plus à l'aise dans la gestion des différentes phases et dans la commutation 
entre les différents schémas cognitifs sollicités. Ensuite, la temporalité du processus met en évidence une pondération variable du triptyque faisabilité/désirabilité/viabilité de Tim Brown. L'approche de Tim Brown doit être modulée par l'avancement dans le projet (cf. Figure 5). En effet si chacun des aspects de faisabilité, viabilité ou désirabilité doit être considéré lors de chaque phase du projet, ces dernières sollicitent plus un élément du triptyque que les trois autres selon l'avancement du projet. Ainsi l'aspect désirabilité est prépondérant dans les deux premières phases avant de s'effacer devant la technologie prépondérante dans les trois dernières phases. La technologie intervient à deux niveaux : en tant que vecteur de faisabilité unitaire (phases 3 et 4) puis ensuite d'un point de vue plus industriel dans son acceptation de génie industriel par la production de volume (phase 5). L'aspect de la viabilité est assez forte dans la phase deux dans sa dimension économie globale puis dans la phase 5 principalement dans son aspect marketing. PEOPLE (DESIRABILITY)

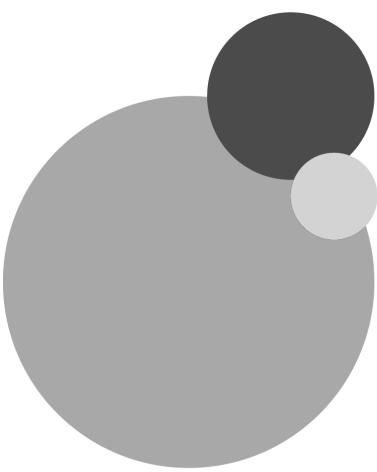

Phase 1-2
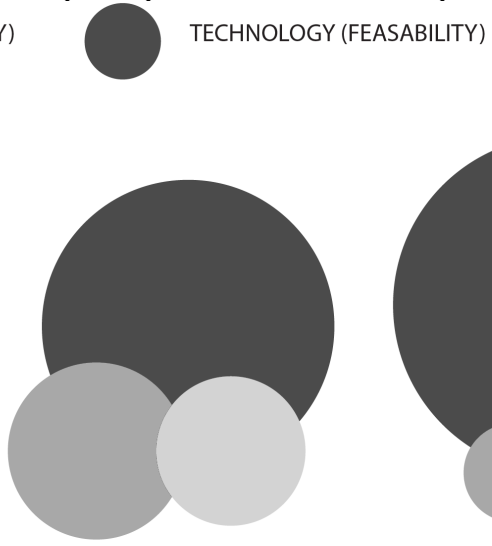

Phase 3

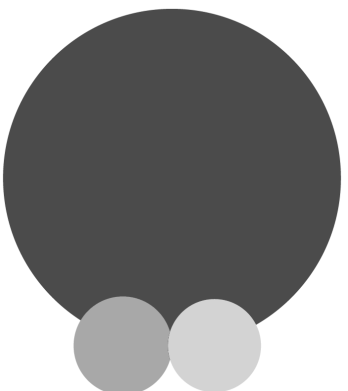

Phase 4
BUSINESS (VIABILITY)

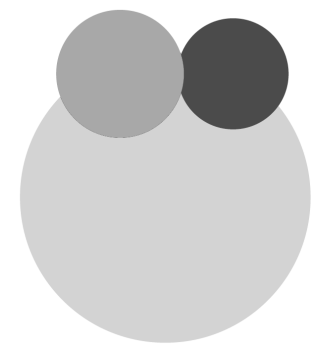

Phase 5

Figure 5 : Introduction de la temporalité dans le formalisme de Tim Brown.

Ce travail de la Figure 5 est en total cohérence avec celui de la Figure 2 et permet de mettre en évidence une adéquation logique entre les différents métiers de l'ingénierie et du marketing, du management et les différentes étapes de ce projet. Nous allons développer cet aspect dans la dernière partie de cet article.

\section{LES STYLES D’APPRENTISSAGE}

En tant qu'approche opérationnelle, notre structuration du Design Thinking peut s'intégrer facilement aussi bien au niveau industriel dans les différents systèmes organisationnels qu'au niveau académique dans les différents cursus d'enseignement général ou plus spécialisé (ingénierie, management, marketing, design, arts,...).

Comme le montre la Figure 4, 4 grandes familles de capacités sont mises en action lors du processus : capacités de raisonner sur des expériences concrètes (Ressentir), capacité de réflexion et d'observation (Voir), capacité à conceptualiser de manière abstraite (Penser) et capacité à expérimenter (Faire). Ces quatre capacités ont été identifiées par David Kolb et Roger Fry en 1975 et synthétisées sous forme de styles d'apprentissage (Kolb 1976). Si personne ne maîtrise de manière égale ces quatre dimensions, toute personne peut en maîtriser deux principales ce qui aboutit aux schémas cognitifs d'apprentissage suivant : convergence, divergence, assimilation et accommodation. Si on met cette classification par modes d'apprentissage en regard du Design Thinking en tant que management du projet, on s'aperçoit que chaque corps de métier a naturellement sa place dans le dispositif d'innovation comme le montre aussi bien le modèle de Buxton modifié que la mise en temporalité de la théorie de Tim Brown. Ainsi le Design Thinking transcende la notion du designer dont il n'est pas l'apanage.

\section{LE DESIGN THINKING, APPROCHE MANAGERIALE DE L'INNOVATION}

Le Design Thinking est une approche managériale de l'innovation enracinée dans des pratiques issues de la $1^{\text {ère }}$ révolution industrielle et développées depuis avec succès. Particulièrement pertinente dans une époque qui cherche un nouveau modèle de management adapté à la globalisation multipolaire et 
aux révolutions socio-techniques diffusées en réseau, cette pensée commence à trouver des espaces pour être développée, enseignée et pratiquée.

\section{IMPLICATIONS POUR LES PRATICIENS}

Le Design Thinking suppose de modifier en profondeur ses pratiques organisationnelles et de mettre en place un processus progressif qui suppose de :

- Diversifier ses équipes, socialement, techniquement, fonctionnellement et culturellement.

- Adopter un mode projet transversal avant même le lancement, respecter les temps de phase et les calendriers.

- Mettre en place un système de veille interne et externe accessible par moteur de recherche.

- Partager les informations sur une plateforme collaborative la plus simple possible.

- Echanger lors d'un cycle de temps forts en équipe, formations, séminaires, journées thématiques, déplacements et voyages d'étude.

- Délivrer le retour d'expérience du projet auprès de tous ses acteurs et ses parties prenantes. Pour ce faire, il est recommandé de sélectionner un premier projet «monstre » ou prototype de l'approche, accompagné par un designer adepte du design Thinking. Ensuite de sélectionner un échantillon diversifié de salariés pouvant faire équipe autour de ce type de projet. Il est alors impératif de dégager un temps réservé et de le valoriser afin de faire « école » au sein de l'organisation. Enfin, de tenir les principaux managers informés durant la poursuite de l'expérience et de les associer au bilan en fin de projet. Alors, le Design Thinking pourra commencer de s'intégrer dans les pratiques « maison ».

Ainsi par son approche centrée sur l'humain, par sa capacité à intégrer la pluridisciplinarité en évitant les démarches en silo en favorisant la collaboration des métiers dans un même espace de projet, le Design Thinking permet d'éviter l'échec de start-up, notamment de type technologiques, et facilite la réussite de projets de type « création de services » ou d'organisations sociales, voire renouveler le développement de produits et de services.

\section{BIBLIOGRAPHIE}

Brown, T. 2009. Change by Design: How Design Thinking Transforms Organizations and Inspires Innovation. NY: Harper Business.

Buxton, Bill. 2007. Sketching User Experiences: Getting the Design Right and the Right Design. San Francisco, CA, USA: Morgan Kaufmann Publishers Inc.

Kimbell, Lucy. 2011. "Rethinking Design Thinking: Part 1." Design and Culture 3 (3): 285-306.

Kolb, David. 1976. Learning Style Inventory: Technical Manual. Boston: McBer and Company.

Bruce Nussbaum « Design Thinking Is A Failed Experiment. So What's Next? »

(http://www.fastcodesign.com/1663558/design-thinking-is-a-failed-experiment-so-whats-next)

Olivier Witmeur : « Entreprendre \& Innover 2012/4 (n 16) 92 pages Editeur De Boeck Supérieur » Jocelyne Le Bœuf, http://designethistoires.lecolededesign.com/2011/06/de-lobjet-icone-au-designthinking/\#more-1406).

\section{BIOGRAPHIES}

Jean-Patrick Péché est designer industriel senior, consultant, formateur, responsable Design Thinking du Msc in IDEA, cofondateur des entreprises de design Dia Design et Design Utility, et d'une entreprise d'innovation Anonymate. 35 ans de pratique du design industriel, 25 ans d'enseignement du design, 15 ans de consultant d'entreprise. 
Fabien Mieyeville, diplômé de 1 'École Centrale de Lyon en 1998, a obtenu son doctorat en électronique dans la même institution en 2001. Maître de Conférences à l'École Centrale Lyon et chercheur à l'Institut des Nanotechnologies de Lyon depuis 2001, il a soutenu son habilitation à diriger les recherches en 2012. Ses thématiques de recherche englobent les systèmes embarqués distribués communicants, les méthodologies de conception hiérarchique de systèmes hétérogènes, les living labs et la conduite de l'innovation par le Design Thinking et l'effectuation. Il est par ailleurs responsable du groupe de recherche associé au Programme IDEA.

Renaud Gaultier est responsable et co-fondateur du programme IDEA au sein de l'Alliance Ecole Centrale Lyon - EMLYON. Diplômé de l'EDHEC en 1987, du M2 Recherche Management des Organisations IAE - Paris 1 - Sorbonne - HEC 2009. Fondateur et associé d'entreprises innovantes, plasticien, créateur de programmes dans l'enseignement supérieur. 